\title{
Comparison of atazanavir/ritonavir and darunavir/ritonavir based antiretroviral therapy for antiretroviral naïve patients
}

Tony Antoniou ${ }^{1,2,3^{*}}$, Leah Szadkowski ${ }^{4}$, Sharon Walmsley ${ }^{3,4}$, Curtis Cooper ${ }^{5}$, Ann N. Burchell ${ }^{1,2,3}$, Ahmed M. Bayoumi ${ }^{1,3}$, Julio S. G. Montaner ${ }^{6,7}$, Mona Loutfy ${ }^{3,8,9}$, Marina B. Klein ${ }^{10}$, Nima Machouf ${ }^{11}$, Christos Tsoukas $^{10}$, Alexander Wong ${ }^{12}$, Robert S. Hogg ${ }^{6,13}$, Janet Raboud ${ }^{3,4}$ and The Canadian Observational Cohort (CANOC) collaboration

\begin{abstract}
Background: Atazanavir/ritonavir and darunavir/ritonavir are common protease inhibitor-based regimens for treating patients with HIV. Studies comparing these drugs in clinical practice are lacking.

Methods: We conducted a retrospective cohort study of antiretroviral naïve participants in the Canadian Observational Cohort (CANOC) collaboration initiating atazanavir/ritonavir- or darunavir/ritonavir-based treatment. We used separate Fine and Gray competing risk regression models to compare times to regimen failure (composite of virologic failure or discontinuation for any reason). Additional endpoints included virologic failure, discontinuation due to virologic failure, discontinuation for other reasons, and virologic suppression.

Results: We studied 222 patients treated with darunavir/ritonavir and 1791 patients treated with atazanavir/ritonavir. Following multivariable adjustment, there was no difference between darunavir/ritonavir and atazanavir-ritonavir in the risk of regimen failure (adjusted hazard ratio $0.76,95 \% \mathrm{Cl} 0.56$ to 1.03) Darunavir/ritonavir-treated patients were at lower risk of virologic failure relative to atazanavir/ritonavir treated patients (aHR $0.50,95 \% \mathrm{Cl} 0.28$ to 0.91 ), findings driven largely by high rates of virologic failure among atazanavir/ritonavir-treated patients in the province of British Columbia. Of 108 discontinuations due to virologic failure, all occurred in patients starting atazanavir/ritonavir. There was no difference between regimens in time to discontinuation for reasons other than virologic failure (aHR 0.93; 95\% Cl 0.65 to 1.33 ) or virologic suppression (aHR $0.99,95 \% \mathrm{Cl} 0.82$ to 1.21 ).
\end{abstract}

Conclusions: The risk of regimen failure was similar between patients treated with darunavir/ritonavir and atazanavir/ ritonavir. Although darunavir/ritonavir was associated with a lower risk of virologic failure relative to atazanavir/ritonavir, this difference varied substantially by Canadian province and likely reflects regional variation in prescribing practices and patient characteristics.

Keywords: HIV, Antiretroviral therapy, Protease inhibitor, Atazanavir, Darunavir

\footnotetext{
* Correspondence: tantoniou@smh.ca

'Keenan Research Centre, Li Ka Shing Knowledge Institute, St. Michael's

Hospital, Toronto, ON, Canada

2Department of Family and Community Medicine, St. Michael's Hospital,

Toronto, ON, Canada

Full list of author information is available at the end of the article
} 


\section{Background}

Protease inhibitors remain important options for the treatment of HIV infection $[1,2]$. When administered with low doses of ritonavir, protease inhibitors impose a high genetic barrier against the selection of drug-resistant variants of HIV and are therefore especially reliable options for patients for whom poor antiretroviral adherence is anticipated $[3,4]$. Because of once-daily dosing and low rates of gastrointestinal adverse effects relative to other members of their class $[5,6]$, ritonavir-boosted atazanavir and darunavir were, until recently, designated as 'preferred' protease inhibitor-based options for the treatment of antiretroviral naïve patients in the United States Depatment of Health and Human Services (DHHS) HIV treatment guidelines [2]. However, atazanavir/ritonavir was reclassified as an 'alternative' to darunavir/ritonavir in the most recent iteration of the DHHS guidelines [2] based on high rates of discontinuation due to toxicity among patients treated with atazanavir/ritonavir in ACTG 5257, a randomized trial comparing the efficacy of atazanavir/ritonavir-, darunavir/ritonavir- and raltegravir-based therapy [7].

Despite these findings, questions remain about the comparative effectiveness of atazanavir/ritonavir and darunavir/ritonavir in clinical practice. Although randomized trials are essential for generating evidence about efficacy required to inform clinical guidelines, individuals enrolled in these studies are often not representative of patients treated in routine care $[8,9]$. Observational studies can address this limitation by providing evidence of the comparative effectiveness and tolerability of different treatment regimens in patients who are treated in clinical practice. Although observational studies comparing the tolerability of atazanavir/ritonavir and darunavir/ritonavir have been conducted, no such studies have specifically compared the effectiveness of these drugs [10-12]. Accordingly, we conducted a retrospective cohort study comparing the effectiveness and durability of atazanavir/ritonavir- and daruanavir/ritonavir-based regimens among antiretroviral naïve patients enrolled in a longitudinal Canadian cohort study.

\section{Methods}

\section{Study population}

The Canadian Observational Cohort (CANOC) collaboration is a multisite cohort study of antiretroviral-naïve HIV positive patients initiating combination antiretroviral therapy (cART) after January 1, 2000 [13]. The collaboration currently includes 8 participating cohorts from Ontario, Quebec and British Columbia. Criteria for inclusion into CANOC were documented HIV infection, residence in Canada, age 18 years and older, initiation of a first antiretroviral regimen comprised of at least three individual agents, and at least one HIV-1 RNA viral load and CD4 count measurement within one year prior to treatment initiation. Antiretroviral data collection methods vary by site, and include abstraction from patient charts and linkage with provincial prescription databases. Nonnominal data were submitted from each participating site to the coordinating center in Vancouver, British Columbia, Canada.

Participants were eligible for inclusion in this analysis if they initiated atazanavir/ritonavir- or darunavir/ritonavir-based antiretroviral therapy, did not have a viral load less than or equal to 200 copies $/ \mathrm{mL}$ at or before cART initiation and had at least one follow-up viral load measurement available after treatment initiation. We excluded patients who initiated atazanavir without concomitant ritonavir.

\section{Outcome measures}

The primary outcome of the study was time to regimen failure, defined as a composite of virologic failure and discontinuation for any reason [Table 1, A (includes B1) or B2]. Similar to ACTG 5257, we defined virologic failure as a viral load $>1000$ copies/mL at or after week 16 but before week 24 , or a viral load $>200$ copies/mL at or after week 24 (A) [7]. Patients who never suppressed or who suppressed and subsequently rebounded were included in this definition. We defined discontinuation as stopping atazanavir or darunavir for more than 60 days. We did not consider changes to the nucleoside backbone, ritonavir, or the addition of other antiretroviral medications as discontinuations. All discontinuations were hierarchically classified as due to virologic failure (B1)or other reasons (B2). Patients who died were considered to have met a competing risk; otherwise patients were censored at the first occurrence of a gap in viral load measurements exceeding two years, the last recorded antiretroviral stop date if followed by a viral load $\leq 50$ copies $/ \mathrm{mL}$, or the last available viral load measurement.

Table 1 Outcome and Competing Risk Definitions

\begin{tabular}{|c|c|c|}
\hline Outcome & Definition & Competing Risks \\
\hline \multicolumn{3}{|l|}{ Primary Analysis } \\
\hline Regimen Failure & $\begin{array}{l}\text { First A (includes B1) } \\
\text { or B2 }\end{array}$ & death \\
\hline \multicolumn{3}{|l|}{ Secondary Analyses } \\
\hline Virologic Failure & First A (includes B1) & B2, death \\
\hline $\begin{array}{l}\text { Discontinuation Due to } \\
\text { Virologic Failure }\end{array}$ & First B1 & B2, death \\
\hline $\begin{array}{l}\text { Discontinuation Due to } \\
\text { Other Reasons }\end{array}$ & First B2 & A (includes B1), death \\
\hline Virologic Suppression & First C & B, death \\
\hline $\begin{array}{l}\text { A. Virologic Failure } \\
\text { B. Discontinuation (hierarc } \\
\text { B2: Due to other reasons) } \\
\text { C. Virologic Suppression }\end{array}$ & Illy classified as B1: Du & to virologic failure, or \\
\hline
\end{tabular}


We conducted several secondary analyses (Table 1). First, we examined time to virologic failure (A) and time to discontinuations due to virologic failure (B1) separately, considering death and discontinuations for other reasons (B2) as competing risks. Next, in the absence of specific adverse event data, we examined time to discontinuation of either darunavir or atazanavir for reasons other than virologic failure (B2). Such discontinuations may have occurred because of toxicities attributable to the protease inhibitor. Individuals who experienced virologic failure (A) and those who died were considered to have met a competing risk. Finally, we examined time to virologic suppression $(C)$, defined as time to the first of at least two consecutive viral load measurements below 50 copies/mL at least 30 days apart. Patients who died or discontinued atazanavir or darunavir for any reason (B) were considered to have met a competing risk. We used the same censoring rules as in the primary analysis in all secondary analyses.

\section{Statistical analysis}

All analyses were conducted with SAS 9.4 (SAS Institute, Cary, North Carolina, USA) and R 3.3.1 (R Development Core Team, Vienna, Austria). We compared baseline characteristics between the two regimen groups using chi-square tests for categorical variables and Wilcoxon rank sum tests for continuous variables. For each outcome, cumulative incidence functions taking into account competing risks were determined by regimen and compared using Gray's Test for Equality [14].

We used multivariable Fine and Gray competing risk regression [15] to estimate the association between treatment with darunavir/ritonavir relative to atazanavir/ritonavir and each outcome. We adjusted our models for age, sex and men who have sex with men (MSM) status, race, baseline viral load and CD4 count, calendar year of treatment initiation, nucleoside analogue backbone (emtricitabine/ tenofovir versus other backbones), Canadian province of residence, and history of injection drug use (IDU). Because of collinearity beween IDU and co-infection with hepatitis $\mathrm{C}$, we adjusted our models for the former variable only. For covariates with large amounts of missing data, separate categories for missing were created when these variables were included in the regression models.

We conducted several sensitivity analyses to test the robustness of our findings. First, we conducted analyses by provincial subgroup (British Columbia vs Ontario/ Quebec) because of regional differences in prescribing patterns and characteristics of CANOC participants [13]. Specifically, antiretroviral treatment guidelines in British Columbia recommended efavirenz or atazanavir/ ritonavir as first-line regimens during our study period, favouring the latter for patients who use drugs or with mental health illness and preserving darunavir/ritonavir for treatment failures. In contrast, no such recommendations were in place in Ontario and Quebec during the study period. Further, participants in British Columbia differ from those in Ontario and Quebec in important ways, including mode of HIV acquisition and hepatitis $\mathrm{C}$ coinfection [13]. We therefore reasoned that residual confounding due to associated unmeasured variables such as mental health illness and socioeconomic status could occur. Second, we examined whether outcomes varied by baseline viral load $(\leq 100,000 \mathrm{copies} / \mathrm{mL}$ or $>100,000$ copies $/ \mathrm{mL}$ ). Third, because approximately onequarter of atazanavir-treated patients started treatment between 2004 and 2006, we replicated our analyses, restricting to patients who started treatment on or after January 1, 2010. Fourth, patients who had a viral load of $\leq 50$ copies $/ \mathrm{mL}$ and who were switched to a single table regimen were re-defined as having discontinued treatment for simplification and included as competing risks. Finally, we replicated our analyses following multiple imputation for missing values of the race, IDU and MSM variables. To our knowledge, no methods currently exist to impute data specifically for Fine \& Gray competing risk regression models, and so a substantive model compatible version of fully conditional specification was used to impute data intended for cause-specific competing risk regression models [16]. For each outcome, all covariates used in the original multivariable models were used to impute missing values five different times. Fine \& Gray models were then run on each imputed dataset and the resulting parameter estimates and variances were combined using Rubin's rules $[17,18]$.

\section{Results}

During the study period, we identified 1791 eligible patients whose first cART regimen included atazanavir/ritonavir and 222 eligible patients whose first regimen included darunavir/ritonavir. Patients treated with atazanavir/ritonavir were more likely to be female $(19 \%$ vs. $13 \% ; p=0.02)$, co-infected with hepatitis C (30\% vs. $13 \% ; p<0.0001)$ and report injection drug use as a risk factor for HIV infection (29\% versus 9\%; $p<0.0001$ ) (Table 2). These differences were mitigated when restricting comparisons to participants from Ontario and Quebec only. The median (interquartile range, IQR) duration of follow-up was $3.5(1.6,5.4)$ years and 1.5 $(0.7,2.3)$ years in the atazanavir/ritonavir and darunavir/ ritonavir treated participants respectively (Table 2 ).

The cumulative incidence of regimen failure (A or B2) one year following the initiation of treatment was 0.29 (95\% confidence interval (CI) 0.27 to 0.31 ) for patients initiating atazanavir/ritonavir and 0.21 (95\% CI 0.15 to 0.27 ) for those starting darunavir/ritonavir ( $p<0.01$, Fig. 1a and b). A total of 43 patients experienced a competing risk of death. Following multivariable adjustment, there was no difference 
Table 2 Demographic and clinical characteristics at initiation of combination antiretroviral therapy by regimen ${ }^{a}$

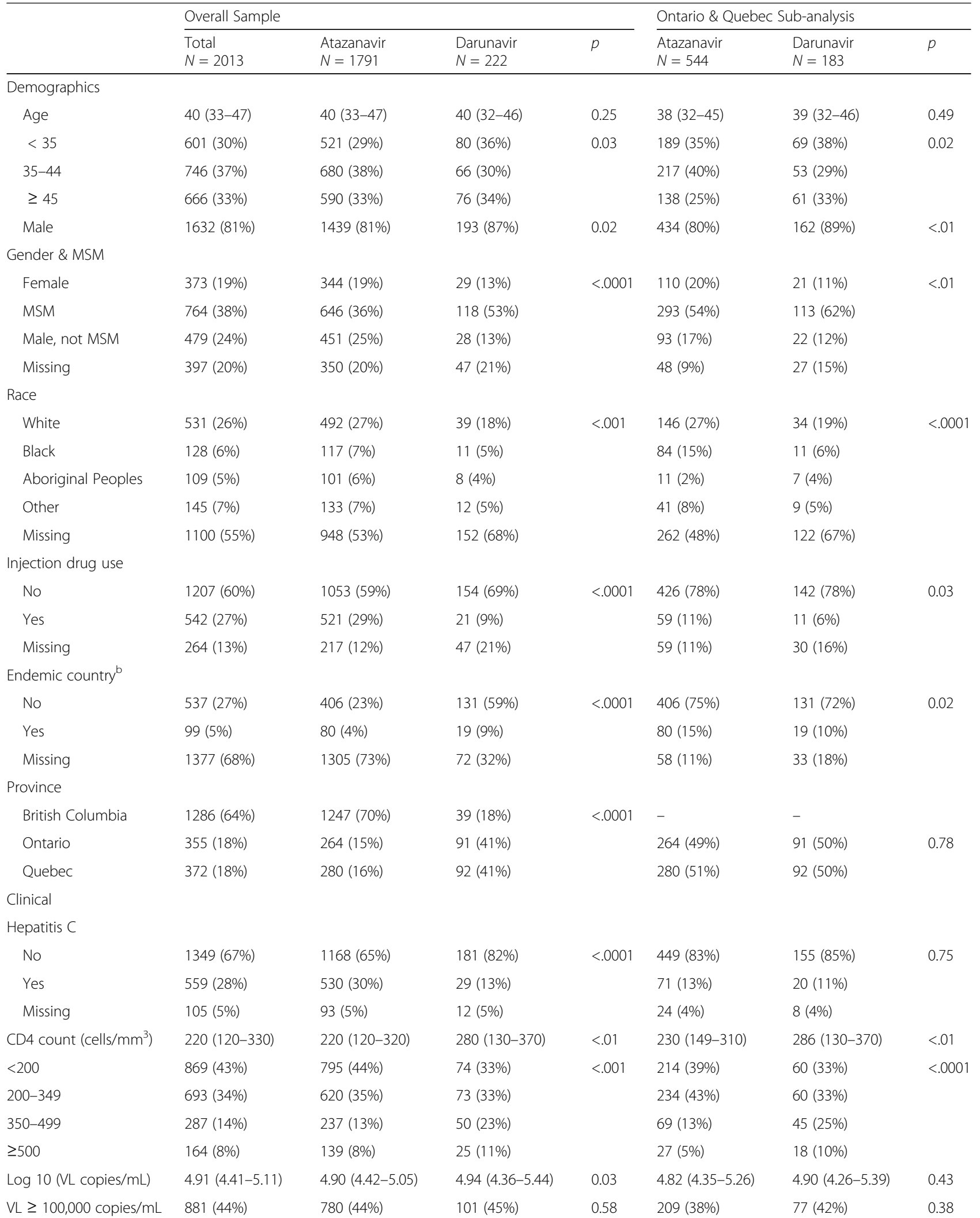


Table 2 Demographic and clinical characteristics at initiation of combination antiretroviral therapy by regimen ${ }^{\text {(Continued) }}$

\begin{tabular}{|c|c|c|c|c|c|c|c|}
\hline Year of CART initiation & 2009 (2007-2010) & 2008 (2006-2010) & 2011 (2010-2011) & $<.0001$ & 2008 (2007-2009) & 2010 (2010-2011) & $<.0001$ \\
\hline 2003-2006 & $474(24 \%)$ & $473(26 \%)$ & $1(0 \%)$ & $<.0001$ & $121(22 \%)$ & $1(1 \%)$ & $<.0001$ \\
\hline 2007-2009 & 799 (40\%) & 767 (43\%) & $32(14 \%)$ & & 307 (56\%) & $32(17 \%)$ & \\
\hline 2010-2012 & $740(37 \%)$ & $551(31 \%)$ & 189 (85\%) & & $116(21 \%)$ & $150(82 \%)$ & \\
\hline \multicolumn{8}{|l|}{ First NRTIs } \\
\hline $3 \mathrm{TC} / \mathrm{ABA}$ & 499 (25\%) & $441(25 \%)$ & $58(26 \%)$ & $<.0001$ & $259(48 \%)$ & $53(29 \%)$ & $<.0001$ \\
\hline 3TC/TDF & $285(14 \%)$ & $284(16 \%)$ & $1(0 \%)$ & & $36(7 \%)$ & $1(1 \%)$ & \\
\hline FTC/TDF & $1172(58 \%)$ & 1009 (56\%) & $163(73 \%)$ & & $230(42 \%)$ & $129(70 \%)$ & \\
\hline Other & $57(3 \%)$ & $57(3 \%)$ & $0(0 \%)$ & & $19(3 \%)$ & $0(0 \%)$ & \\
\hline \multicolumn{8}{|l|}{ Follow up } \\
\hline Years of Follow up & $3.10(1.45-5.16)$ & $3.50(1.63-5.38)$ & $1.48(0.69-2.25)$ & $<.0001$ & $3.73(2.20-5.32)$ & $1.68(0.86-2.46)$ & $<.0001$ \\
\hline VLs per year & $4.67(3.61-6.21)$ & $4.68(3.63-6.16)$ & $4.50(3.55-6.56)$ & 0.71 & $3.88(3.12-4.76)$ & $4.32(3.44-5.76)$ & $<.001$ \\
\hline $0-3$ & $274(14 \%)$ & $246(14 \%)$ & $28(13 \%)$ & 0.43 & $121(22 \%)$ & $26(14 \%)$ & $<.001$ \\
\hline $3-6$ & 1187 (59\%) & $1062(59 \%)$ & $125(56 \%)$ & & $358(66 \%)$ & $115(63 \%)$ & \\
\hline$>6$ & $552(27 \%)$ & $483(27 \%)$ & 69 (31\%) & & $65(12 \%)$ & $42(23 \%)$ & \\
\hline
\end{tabular}

MSM men who have sex with men, $V L$ viral load, $C A R T$ combination antiretroviral therapy, NRTI nucleoside reverse transcriptase, $3 T C$ lamivudine, $A B C$ abacavir, TDF tenofovir, FTC emtricitabine

a Medians (IQRs) are presented for continuous variables and frequencies (percent) for categorical variables

b Indicates patients who have immigrated to Canada from a country with a high prevalence of HIV

between darunavir/ritonavir and atazanavir-ritonavir in the risk of regimen failure (adjusted hazard ratio 0.76, 95\% CI 0.56 to 1.03 ) (Table 3$)$. This finding was similar in sensitivity analyses (Table 4).

The one-year cumulative incidence of virologic failure (A) was 0.09 (95\% CI 0.08 to 0.10 ) for patients starting atazanavir/ritonavir and 0.04 (95\% CI 0.02 to 0.08$)$ for those starting darunavir/ritonavir ( $p=0.02$, Fig. 1a and $\mathrm{b}$ ). Forty-three patients died prior to virologic failure. Following multivariable adjustment, the risk of virologic failure was lower in darunavir/ritonavir-treated patients (adjusted hazard ratio $0.50,95 \%$ CI 0.28 to 0.91 ) (Table 3 ). However, this finding was driven primarily by high failure rates among atazanavir/ritonavir-treated patients in the province of British Columbia. In a sensitivity analysis by Canadian province, there was no difference in the risk of virologic failure among participants in Ontario and Quebec (adjusted hazard ratio $0.90,95 \%$ CI 0.44 to 1.86 ) (Table 4). In the analysis of participants from British Columbia, the point estimate of the adjusted hazard ratio of virologic failure associated with darunavir was similar (adjusted hazard ratio $=0.41,95 \% \mathrm{CI}(0.09,1.78)$ ) to that from the analysis of the entire cohort but the finding was not statistically significant due to the infrequent use of darunavir in this province (Table 4).

The cumulative incidence of discontinuation due to virologic failure (B1) in atazanavir/ritonavir and darunavir/ritonavir-treated patients was 0.01 (95\% CI 0.01 to 0.02 ) and 0.00 , respectively ( $p=0.01$, Fig. $1 \mathrm{a}$ and $\mathrm{b})$. The lack of events in darunavir/ritonavir treated patients resulted in adjusted hazard ratios of 0.00 for darunavir/ ritonavir vs. atazanavir/ritonavir when modelling this outcome.

The cumulative incidence of discontinuation for reasons other than virologic failure (B2) was 0.2 (95\% CI 0.18 to 0.22 ) for patients initiating atazanavir/ritonavir and 0.17 (95\% CI 0.12 to 0.22$)$ for patients initiating darunavir/ritonavir ( $p=0.16$, Fig. $1 \mathrm{a}$ and $\mathrm{b})$. Forty-three patients experienced a competing risk of death. There was no difference between darunavir/ritonavir and atazanavir/ritonavir in the risk of discontinuation for reasons other than virologic failure after adjusting for confounding variables (adjusted hazard ratio $0.93,95 \%$ CI 0.65 to 1.33) (Table 3). These results were similar in sensitivity analyses (Table 4).

The cumulative incidence of virologic suppression (C) at 1 year after treatment initiation was 0.73 (95\% CI 0.70 to 0.75$)$ for participants whose first regimen included atazanavir/ritonavir and 0.73 (95\% CI 0.66 to 0.79$)$ for those whose first regimen included darunavir/ritonavir ( $p=0.62$, Fig. $1 \mathrm{c}$ and d). Twenty-four patients died prior to achieving virologic suppression. Following multivariable adjustment, there was no difference in the time to virologic suppression (adjusted hazard ratio 0.99, 95\% CI 0.82 to 1.21 ) according to treatment regimen (Table 3 ). Results were similar in sensitivity analyses (Table 4).

In sensitivity analyses of patients who initiated treatment on or after January 1, 2010, the adjusted hazards ratios for treatment group were very similar to those of the main analysis considering patients starting on or after January 1, 2003 (Table 4). However, the estimates were less precise because of the smaller sample size. 

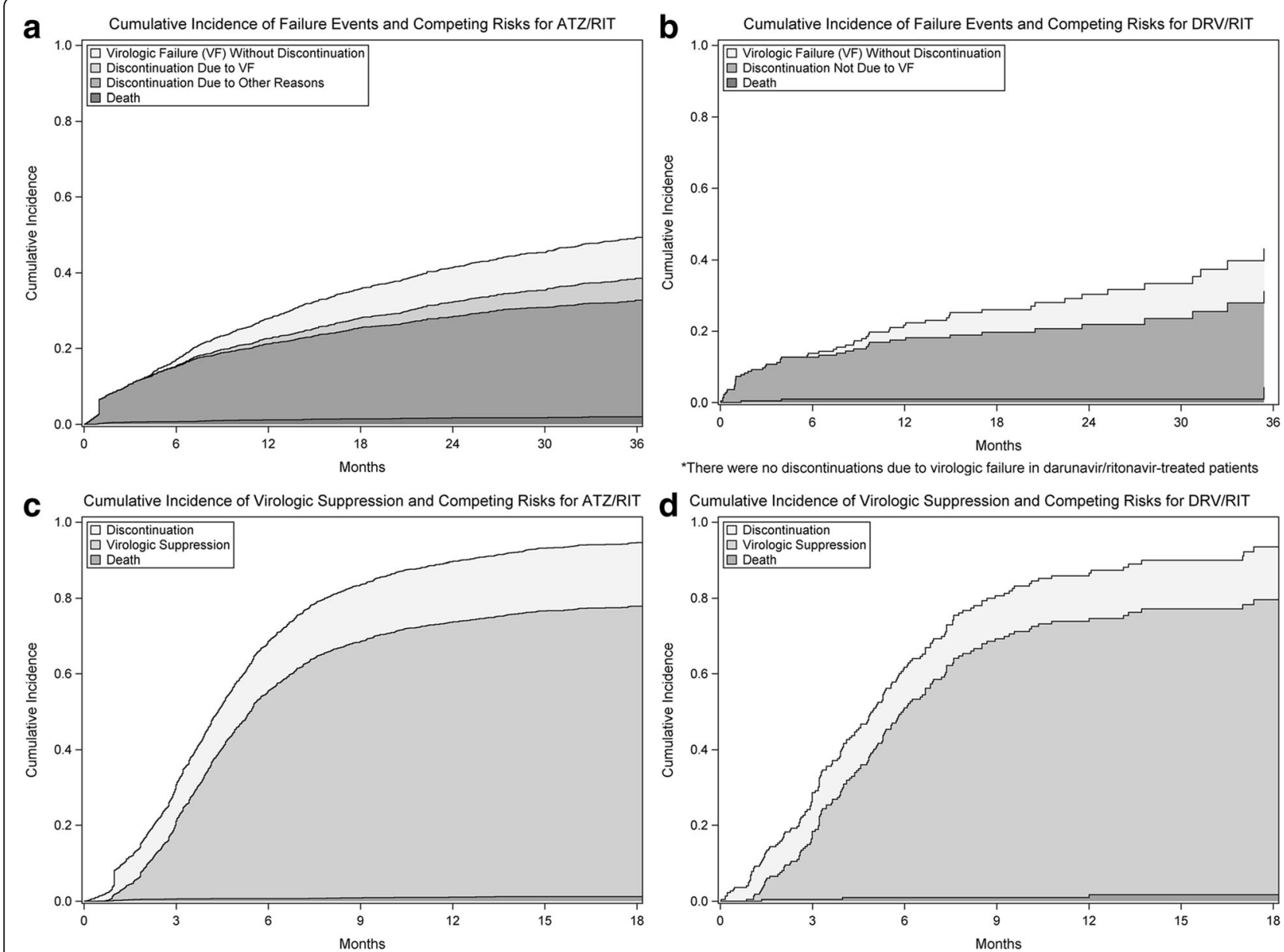

Fig. 1 Cumulative incidence functions of time to events and competing risks

\section{Discussion}

In our analysis of more than two thousand antiretroviral naïve patients from three Canadian provinces, patients initiating darunavir/ritonavir were at a lower risk of virologic failure (A) and subsequent discontinuations due to virologic failure (B1) than patients treated with atazanavir/ritonavir. In contrast, we observed no differences in time to regimen failure (A or B2), virologic suppression $(C)$ or to discontinuation for reasons other than virologic failure (B2).

Our findings differ from those of ACTG 5257, a randomized trial comparing darunavir/ritonavir, atazanavir/ ritonavir- and raltegravir-based antiretroviral therapy [7]. Specifically, this trial found a higher incidence of tolerability discontinuation among patients randomized to atazanavir/ritonavir, mediated primarily by participant-driven regimen change for jaundice or hyperbilirubinemia and non-hepatobiliary gastrointestinal side effects. Although we did not have data regarding the exact reasons for discontinuation, we found no difference in the risk of discontinuations for reasons other than virologic failure (B2), an outcome which includes toxicity-driven discontinuation. In a sensitivity analysis, there was no difference in the risk of discontinuations for reasons other than (i) virologic failure or (ii) simplification, an outcome that may more closely approximate toxicity-driven discontinuation. The discrepancy between the results of our analysis and those of the ACTG 5257 trial may be due in part to differences in the calendar year periods of atazanavir and darunavir initiation in our study. Because atazanavir-treated patients started treatment approximately three years earlier than darunavir-treated patients, with approximately onequarter starting between 2004 and 2006, we speculated that discontinuations due to jaundice or asymptomatic hyperbilirubinemia were tempered by a lack of potent, tolerable treatment alternatives during this period. Most notably, $70 \%$ of patients starting atazanavir/ritonavir resided in British Columbia, a province where the epidemic is driven primarily by injection drug use, thereby potentially deterring clinicians from switching patients to regimens with lower barriers to resistance or higher rates of gastrointestinal side effects. In contrast, ACTG 5257 
Table 3 Multivariable Fine and Gray models of time to events

\begin{tabular}{|c|c|c|c|c|c|c|c|c|c|c|}
\hline & \multicolumn{2}{|c|}{$\begin{array}{l}\text { Virologic Failure or } \\
\text { Other Discontinuation }\end{array}$} & \multicolumn{2}{|l|}{ Virologic Failure } & \multicolumn{2}{|c|}{$\begin{array}{l}\text { Discontinuation due to } \\
\text { Virologic Failure }\end{array}$} & \multicolumn{2}{|l|}{$\begin{array}{l}\text { Other } \\
\text { Discontinuation }\end{array}$} & \multicolumn{2}{|c|}{ Virologic Suppression } \\
\hline & aHR (95\% Cl) & $p$ & aHR $(95 \% \mathrm{Cl})$ & $p$ & aHR (95\% Cl) & $p$ & aHR (95\% Cl) & $p$ & aHR $(95 \% \mathrm{Cl})$ & $p$ \\
\hline DRV/RIT & $0.76(0.56,1.03)$ & 0.08 & $0.50(0.28,0.91)$ & 0.02 & $0.00(0.00,0.00)$ & $<.0001$ & $0.93(0.65,1.33)$ & 0.68 & $0.99(0.82,1.21)$ & 0.96 \\
\hline FTC/TEN & $1.02(0.86,1.21)$ & 0.84 & $1.30(0.99,1.70)$ & 0.06 & $1.41(0.87,2.28)$ & 0.16 & $0.87(0.70,1.08)$ & 0.20 & $0.99(0.87,1.12)$ & 0.82 \\
\hline Age (per 10y) & $0.89(0.83,0.95)$ & $<.001$ & $1.02(0.91,1.15)$ & 0.70 & $0.89(0.73,1.09)$ & 0.27 & $0.86(0.79,0.94)$ & $<.001$ & $1.04(0.98,1.10)$ & 0.17 \\
\hline \multicolumn{11}{|l|}{ Gender \& MSM } \\
\hline Female & Ref. & & Ref. & & Ref. & & Ref. & & Ref. & \\
\hline MSM & $0.68(0.55,0.84)$ & $<.001$ & $0.73(0.51,1.03)$ & 0.08 & $0.46(0.24,0.89)$ & 0.02 & $0.79(0.61,1.03)$ & 0.08 & $1.20(1.00,1.45)$ & 0.05 \\
\hline Male, not MSM & $0.72(0.59,0.88)$ & $<.01$ & $0.90(0.64,1.25)$ & 0.51 & $0.70(0.42,1.17)$ & 0.17 & $0.76(0.59,0.98)$ & 0.03 & $1.06(0.87,1.28)$ & 0.57 \\
\hline Missing & $0.74(0.56,0.98)$ & 0.03 & $0.80(0.47,1.35)$ & 0.40 & $0.64(0.27,1.55)$ & 0.33 & $0.83(0.60,1.15)$ & 0.26 & $1.13(0.90,1.42)$ & 0.31 \\
\hline \multicolumn{11}{|l|}{ Race } \\
\hline White & Ref. & & Ref. & & Ref. & & Ref. & & Ref. & \\
\hline Black & $0.89(0.64,1.23)$ & 0.47 & $1.41(0.88,2.26)$ & 0.15 & $1.18(0.47,2.95)$ & 0.72 & $0.64(0.40,1.04)$ & 0.07 & $1.16(0.88,1.54)$ & 0.29 \\
\hline Aboriginal Peoples & $1.39(1.05,1.85)$ & 0.02 & $1.14(0.72,1.82)$ & 0.57 & $1.46(0.71,2.99)$ & 0.30 & $1.32(0.91,1.89)$ & 0.14 & $0.68(0.51,0.92)$ & 0.01 \\
\hline Other & $0.89(0.67,1.18)$ & 0.42 & $1.04(0.68,1.59)$ & 0.86 & $0.79(0.36,1.75)$ & 0.56 & $0.82(0.56,1.19)$ & 0.29 & $1.15(0.94,1.40)$ & 0.17 \\
\hline Missing & $1.17(0.98,1.39)$ & 0.08 & $0.75(0.55,1.01)$ & 0.06 & $0.70(0.43,1.14)$ & 0.15 & $1.38(1.12,1.72)$ & $<.01$ & $0.93(0.80,1.08)$ & 0.35 \\
\hline \multicolumn{11}{|l|}{ IDU } \\
\hline No & Ref. & & Ref. & & Ref. & & Ref. & & Ref. & \\
\hline Yes & $1.68(1.39,2.02)$ & $<.0001$ & $1.46(1.05,2.04)$ & 0.03 & $1.64(0.92,2.94)$ & 0.09 & $1.50(1.20,1.88)$ & $<.001$ & $0.69(0.58,0.82)$ & $<.0001$ \\
\hline Missing & $1.11(0.83,1.49)$ & 0.48 & $1.16(0.68,1.96)$ & 0.58 & $1.10(0.41,2.93)$ & 0.85 & $1.04(0.74,1.45)$ & 0.83 & $0.91(0.72,1.14)$ & 0.40 \\
\hline \multicolumn{11}{|l|}{ Province } \\
\hline$B C$ & Ref. & & Ref. & & Ref. & & Ref. & & Ref. & \\
\hline ON & $0.95(0.77,1.16)$ & 0.60 & $1.11(0.81,1.54)$ & 0.52 & $0.67(0.34,1.31)$ & 0.24 & $0.90(0.69,1.17)$ & 0.44 & $0.97(0.82,1.15)$ & 0.73 \\
\hline QC & $0.82(0.66,1.02)$ & 0.08 & $1.26(0.88,1.80)$ & 0.21 & $1.03(0.51,2.08)$ & 0.93 & $0.68(0.51,0.90)$ & $<.01$ & $1.02(0.86,1.21)$ & 0.83 \\
\hline \multicolumn{11}{|l|}{ Baseline CD4 (per 100) } \\
\hline$<200$ & Ref. & & Ref. & & Ref. & & Ref. & & Ref. & \\
\hline 200-349 & $0.83(0.71,0.96)$ & 0.01 & $0.93(0.73,1.20)$ & 0.58 & $1.16(0.76,1.77)$ & 0.48 & $0.84(0.69,1.02)$ & 0.08 & $1.18(1.04,1.33)$ & $<.01$ \\
\hline $350-499$ & $0.80(0.63,1.01)$ & 0.06 & $0.52(0.33,0.84)$ & $<.01$ & $0.60(0.23,1.56)$ & 0.29 & $1.00(0.76,1.31)$ & 0.99 & $1.35(1.14,1.61)$ & $<.001$ \\
\hline$\geq 500$ & $1.07(0.80,1.45)$ & 0.64 & $0.79(0.44,1.42)$ & 0.44 & $0.95(0.33,2.69)$ & 0.92 & $1.22(0.87,1.71)$ & 0.25 & $1.36(1.08,1.71)$ & $<.01$ \\
\hline Baseline VL $\geq 100,000$ & $1.20(1.05,1.38)$ & $<.01$ & $1.74(1.38,2.20)$ & $<.0001$ & $2.27(1.49,3.48)$ & $<.001$ & $0.95(0.80,1.13)$ & 0.57 & $0.61(0.55,0.68)$ & $<.0001$ \\
\hline \multicolumn{11}{|l|}{ Calendar Year } \\
\hline 2003-2006 & Ref. & & Ref. & & Ref. & & Ref. & & Ref. & \\
\hline 2007-2009 & $0.96(0.79,1.17)$ & 0.69 & $0.68(0.50,0.92)$ & 0.01 & $0.41(0.24,0.71)$ & $<.01$ & $1.11(0.87,1.42)$ & 0.39 & $0.96(0.82,1.12)$ & 0.58 \\
\hline 2010-2012 & $1.12(0.88,1.42)$ & 0.35 & $0.88(0.61,1.28)$ & 0.50 & $0.64(0.33,1.24)$ & 0.19 & $1.03(0.77,1.38)$ & 0.84 & $0.67(0.56,0.81)$ & $<.0001$ \\
\hline
\end{tabular}

AHR adjusted hazard ratio, DRV/r darunavir/ritonavir, ATZ/r atazanavir/ritonavir, FTC emtricitabine, TDF tenofovir, NRTI nucleoside reverse transcriptase inhibitor, $M S M$ men who have sex with men, $V L$ viral load

participants initiated treatment between 2009 and 2011, a period marked by the availability of potent treatment alternatives conducive to supporting participant-driven requests for regimen change. Although sensitivity analyses restricted to participants initiating cART between 2010 and 2012 yielded similar results, we speculate that this reflects a desire to preserve darunavir/ritonavir and other new options as second-line regimens for patients treated with atazanavir/ritonavir in British Columbia.
Our finding of an increased risk of virologic failure among patients receiving atazanavir/ritonavir also contrasts with the results of ACTG 5257, in which no difference was observed in this outcome. A possible explanation relates to inter-study differences in the prevalence and distribution of baseline characteristics known to adversely affect adherence and sustained virologic suppression. Specifically, relative to ACTG 5257 our study population included a greater proportion of patients who had a history of injection drug use $(27 \%$ 
Table 4 Adjusted hazard ratios for darunavir/ritonavir vs. atazanavir/ritonavir from sensitivity analyses

\begin{tabular}{|c|c|c|c|c|c|c|c|c|c|c|c|}
\hline \multirow{2}{*}{\multicolumn{2}{|c|}{ Analysis }} & \multicolumn{2}{|c|}{$\begin{array}{l}\text { Virologic Failure or } \\
\text { Other Discontinuation }\end{array}$} & \multicolumn{2}{|l|}{ Virologic Failure } & \multicolumn{2}{|c|}{$\begin{array}{l}\text { Discontinuations due } \\
\text { to Virologic Failure }\end{array}$} & \multicolumn{2}{|l|}{$\begin{array}{l}\text { Other } \\
\text { Discontinuation }\end{array}$} & \multicolumn{2}{|l|}{$\begin{array}{l}\text { Virologic } \\
\text { Suppression }\end{array}$} \\
\hline & & aHR (95\% Cl) & $p$ & aHR $(95 \% \mathrm{Cl})$ & $p$ & aHR (95\% Cl) & $p$ & aHR $(95 \% \mathrm{Cl})$ & $p$ & $\mathrm{aHR}(95 \% \mathrm{Cl})$ & $p$ \\
\hline 0 & Original Model & $0.76(0.56,1.03)$ & 0.08 & $0.50(0.28,0.91)$ & 0.02 & $0.00(0.00,0.00)$ & $<.0001$ & $0.93(0.65,1.33)$ & 0.68 & $0.99(0.82,1.21)$ & 0.96 \\
\hline \multicolumn{12}{|c|}{1 Subgroup by Province } \\
\hline & ON/QC Only & $0.73(0.51,1.06)$ & 0.10 & $0.90(0.44,1.86)$ & 0.78 & $0.00(0.00,0.00)$ & $<.0001$ & $0.72(0.47,1.11)$ & 0.14 & $0.99(0.78,1.27)$ & 0.96 \\
\hline & BC Only & $0.69(0.34,1.42)$ & 0.32 & $0.41(0.09,1.78)$ & 0.23 & $0.00(0.00,0.00)$ & $<.0001$ & $0.93(0.43,2.04)$ & 0.86 & $0.78(0.46,1.32)$ & 0.35 \\
\hline \multicolumn{12}{|c|}{2 Subgroup by Baseline VL } \\
\hline & $<100,000$ copies/mL & $0.66(0.42,1.03)$ & 0.07 & $0.47(0.18,1.19)$ & 0.11 & $0.00(0.00,0.00)$ & $<.0001$ & $0.82(0.49,1.36)$ & 0.44 & $0.97(0.76,1.23)$ & 0.78 \\
\hline & $\geq 100,000$ copies $/ \mathrm{mL}$ & $0.86(0.56,1.33)$ & 0.49 & $0.54(0.25,1.16)$ & 0.11 & $0.00(0.00,0.00)$ & $<.0001$ & $1.16(0.68,2.00)$ & 0.58 & $1.11(0.81,1.53)$ & 0.51 \\
\hline 3 & 2010-2012 Only & $0.81(0.56,1.18)$ & 0.27 & $0.55(0.26,1.14)$ & 0.11 & $0.00(0.00,0.00)$ & $<.0001$ & $0.96(0.63,1.48)$ & 0.87 & $0.98(0.76,1.26)$ & 0.87 \\
\hline 4 & $\begin{array}{l}\text { Simplification discontinuations } \\
\text { included as competing risks }\end{array}$ & $0.75(0.54,1.03)$ & 0.07 & $0.50(0.28,0.91)$ & 0.02 & $0.00(0.00,0.00)$ & $<.0001$ & $0.92(0.63,1.34)$ & 0.65 & $0.99(0.82,1.21)$ & 0.96 \\
\hline 5 & SMC-FCS Imputation & $0.76(0.56,1.04)$ & 0.08 & $0.5(0.28,0.9)$ & 0.02 & $0.00(0.00,0.00)$ & $<.0001$ & $0.91(0.62,1.34)$ & 0.64 & $0.98(0.8,1.2)$ & 0.84 \\
\hline
\end{tabular}

aHR adjusted hazard ratio, $V L$ viral load

versus $7 \%$ ), were coinfected with hepatitis C (28\% versus $7.8 \%)$ and with baseline CD4 counts below 350 cells/ $\mathrm{mm}^{3}$ (77\% versus $8.8 \%$ ). Notably, these characteristics were disproportionately represented among patients treated with atazanavir/ritonavir in our study, likely predisposing these patients to virologic failure.

Inter-provincial differences in patient characteristics and prescribing practices may also account for disparate findings between our study and ACTG 5257. Specifically, a higher prevalence of injection drug use and hepatitis C was observed among participants in British Columbia relative to Ontario and Quebec. Although we adjusted for injection drug use in our analysis, we lacked data regarding co-existing mental health illness, other substance use and socioeconomic status; residual confounding is therefore possible. Also, as noted earlier, atazanavir/ritonavir was the recommended protease inhibitor for antiretroviral naïve patients in British Columbia during the study period, particularly for patients with co-morbid illness or social circumstances that could predispose them to treatment failure. In contrast, no such recommendations were in place in Ontario and Quebec during the study period. The impact of these selection biases was evident in sensitivity analyses restricted to participants from Ontario and Quebec, in that no differences between regimens were observed for any outcome, including virologic failure.

Strengths of our analysis include the size of the study population, the diversity of the participants in CANOC and the ability to compare clinical outcomes between individuals initiating atazanavir/ritonavir and darunavir/ritonavir in a clinical practice setting. However, several limitations of our study merit emphasis. Most notably, as with all observational studies, our findings may be biased by residual inter-group differences in baseline variables and unmeasured confounders. In addition, as noted earlier, differences in the timing of darunavir and atazanavir availability may have precluded our ability to observe differences in the risk of treatment discontinuation. The nature of antiretroviral data in a retrospective cohort also imposes some limitations when considering discontinuation. Some sites have prescription based data with stop dates calculated from the days supplied by the prescription. These stop dates are inaccurate when a patient is not perfectly adherent. Other sites provide start and stop dates abstracted from patient charts which are subject to recall and documentation errors. Since $70 \%$ of participants who had atazanavir as part of their first regimen resided in British Columbia, where antiretroviral records are prescription-based compared to $18 \%$ of participants who had darunavir as part of their starting regimen, this may have resulted in an overestimate of time to discontinuation among participants on atazanavir. Finally, we lacked data regarding exact reasons for treatment discontinuation.

\section{Conclusions}

In conclusion, darunavir/ritonavir and atazanavir/ritonavir were of similar effectiveness in the treatment of antiretroviral naïve patients. Differences in the risk of virologic failure between darunavir/ritonavir and atazanavir/ritonavir varied substantially by province due to regional differences in prescribing patterns and patient characteristics. Our data provide both a comparison of the effectiveness of these two protease inhibitors in the clinical setting and an illustration of the potential magnitude and impact of selection bias in a cohort study setting.

\section{Abbreviations}

CANOC: Canadian observational cohort: CART: Combination antiretroviral therapy; Cl: Confidence interval; DHHS: Department of health and human services; IDU: Injection drug use; IQR: Interquartile range; MSM: Men who have sex with men 


\section{Acknowledgements}

We would like to thank all of the participants for allowing their information to be a part of the CANOC collaboration.

The CANOC Collaborative Research Centre includes: CANOC Nominated Principal Investigator: Robert Hogg (British Columbia Centre for Excellence in HIV/AIDS, Simon Fraser University).

Principal Investigators: Ann N. Burchell (St. Michael's Hospital, University of Toronto), Curtis Cooper (University of Ottawa, OCS), Deborah Kelly (Memorial University of Newfoundland), Marina Klein (Montreal Chest Institute Immunodeficiency Service Cohort, McGill University), Mona Loutfy (University of Toronto, Maple Leaf Medical Clinic, OCS), Nima Machouf (Clinique Medicale l'Actuel, Université de Montréal), Julio Montaner (British Columbia Centre for Excellence in HIV/AIDS, University of British Columbia), Janet Raboud (University of Toronto, University Health Network, OCS), Chris Tsoukas (McGill University), Stephen Sanche (University of Saskatchewan), Alexander Wong (University of Saskatchewan), Tony Antoniou (St. Michael's Hospital, University of Toronto, Institute for Clinical Evaluative Sciences), Ahmed Bayoumi (St. Michael's Hospital, University of Toronto), Mark Hull (British Columbia Centre for Excellence in HIV/AIDS), Bohdan Nosyk (British Columbia Centre for Excellence in HIV/AIDS, Simon Fraser University). Co-Investigators: Angela Cescon (Northern Ontario School of Medicine), Michelle Cotterchio (Cancer Care Ontario, University of Toronto), Charlie Goldsmith (Simon Fraser University), Silvia Guillemi (British Columbia Centre for Excellence in HIV/AIDS, University of British Columbia), P. Richard Harrigan (British Columbia Centre for Excellence in HIV/AIDS, University of British Columbia), Marianne Harris (St. Paul's Hospital), Sean Hosein (CATIE), Sharon Johnston (Bruyère Research Institute, University of Ottawa), Claire Kendall (Bruyère Research Institute, University of Ottawa), Clare Liddy (Bruyère Research Institute, University of Ottawa), Viviane Lima (British Columbia Centre for Excellence in HIV/AIDS, University of British Columbia), David Marsh (Northern Ontario School of Medicine), David Moore (British Columbia Centre for Excellence in HIV/AIDS, University of British Columbia), Alexis Palmer (British Columbia Centre for Excellence in HIV/AIDS, Simon Fraser University), Sophie Patterson (British Columbia Centre for Excellence in HIV/ AIDS, Simon Fraser University), Peter Phillips (British Columbia Centre for Excellence in HIV/AIDS, University of British Columbia), Anita Rachlis (University of Toronto, OCS), Sean B. Rourke (University of Toronto, OCS), Hasina Samji (British Columbia Centre for Excellence in HIV/AIDS), Marek Smieja (McMaster University), Benoit Trottier (Clinique Medicale l'Actuel, Université de Montréal), Mark Wainberg (McGill University, Lady Davis Institute for Medical Research), Sharon Walmsley (University Health Network, University of Toronto)

Collaborators: Chris Archibald (Public Health Agency of Canada Centre for Communicable Diseases and Infection Control), Ken Clement (Canadian Aboriginal AIDS Network), Monique Doolittle-Romas (Canadian AIDS Society), Laurie Edmiston (Canadian Treatment Action Council), Sandra Gardner (OHTN, University of Toronto, OCS), Brian Huskins (Canadian Treatment Action Council), Jerry Lawless (University of Waterloo), Douglas Lee (University Health Network, University of Toronto, ICES), Renee Masching (Canadian Aboriginal AIDS Network), Stephen Tattle (Canadian Working Group on HIV \& Rehabilitation), Alireza Zahirieh (Sunnybrook Health Sciences (entre).

Analysts and Staff: Claire Allen (Regina General Hospital), Stryker Calvez (SHARE), Guillaume Colley (British Columbia Centre for Excellence in HIV/ AIDS), Jason Chia (British Columbia Centre for Excellence in HIV/AIDS), Daniel Corsi (The Ottawa Hospital Immunodeficiency Clinic, Ottawa Hospital Research Institute), Louise Gilbert (Immune Deficiency Treatment Centre), Nada Gataric (British Columbia Centre for Excellence in HIV/AIDS), Katelyn Merritt (British Columbia Centre for Excellence in HIV/AIDS), Lucia Light (OHTN), David Mackie (The Ottawa Hospital), Costa Pexos (McGill University), Susan Shurgold (British Columbia Centre for Excellence in HIV/AIDS), Leah Szadkowski (University Health Network), Chrissi Galanakis (Clinique Médicale L'Actuel), Benita Yip (British Columbia Centre for Excellence in HIV/AIDS), Jaime Younger (University Health Network), and Julia Zhu (British Columbia Centre for Excellence in HIV/AIDS).

\section{Funding}

CANOC is funded by the Canadian Institutes of Health Research (CIHR) through a Centres Grant (Centres for HIV/AIDS Population Health and Health Services Research [CIHR 02684]), two Operating Grants (HIV/AIDS Priority Announcement [CIHR 134047]; Population and Public Health [CIHR 136882]), and is supported by the CIHR Canadian HIV Trials Network (CTN 242) and a Foundation Grant (Expansion of Antiretroviral Therapy and its Impact on Vulnerable Populations in Canada and Global Settings [CIHR 143342]). JR is supported through an Ontario HIV Treatment Network (OHTN) Chair in Biostatistics. TA is supported by a New Investigator Award from OHTN/CIHR. $A M B$ is supported by the Fondation Baxter and Alma Ricard Chair in Inner City Health. ANB is supported by a CIHR New Investigator Award. CC is supported through an Applied HIV Research Chair from the OHTN. RSH is supported by a University Professorship at Simon Fraser University. MBK is supported by a Chercheur National Career Award from the Fonds de recherche du Québec - Santé (FRQ-S). JSGM is supported by the British Columbia Ministry of Health and by the US National Institutes of Health (R01DA036307). SW is supported through a Applied Health Research Chair from the OHTN.

\section{Availability of data and materials}

The data analysed during the current study are not publicly available due to restrictions from data sharing agreements among our institutions to protect the privacy of study participants.

\section{Authors' contribution}

TA and JR conceived of the study, LS conducted statistical analyses, and TA, $J R$ and LS drafted the manuscript. RSH, JR, ANB, CC, MBK, ML, NM, JSGM and $C T$ were involved in data acquisition. All authors reviewed iterations of the analyses and drafts of the manuscript and approved the final version of the manuscript.

\section{Competing interests}

CC has served on advisory boards for Abbvie and Gilead Sciences. MBK reports grants from Merck and ViiV Healthcare and personal fees for consultancy from ViiV Healthcare, Bristol-Meyers Squibb, and Merck. ML has served on advisory boards and spoken at CME events for Viiv Healthcare, Abbvie, Merck Canada Inc. and Gilead Sciences. NM has been a speaker for Bristol-Meyers Squibb, Merck, and ViiV Healthcare. JSGM is supported with grants paid to his institution by the British Columbia Ministry of Health and by the US National Institutes of Health (R01DA036307). He has also received limited unrestricted funding, paid to his institution, from Abbvie, Bristol-Myers Squibb, Gilead Sciences, Janssen, Merck, and ViiV Healthcare. JR is co-investigator on two projects with in-kind contributions from Merck and Gilead Sciences. SW has served on advisory boards and spoken at CME events for Viiv, Abbvie, Merck, Gilead, Jannsen, and Bristol Meyers Squibb. AW has received honouraria for speaking events and advisory meetings with Gilead Sciences, ViiV Healthcare, Bristol-Myers Squibb, Janssen, Abbvie, and Merck and is an investigator in clinical trials with Gilead Sciences, Merck and ViiV Healthcare. RSH has received funding from Canadian Institutes of Health Research, through a Foundation Award, to support this research and reports personal fees from Gilead Sciences. For the remaining authors no conflicts of interest were declared.

\section{Consent for publication}

Not applicable.

\section{Ethics approval and consent to participate}

Ethics approval of the CANOC collaboration was granted by the Simon Fraser University Research Ethics Board (REB) and the University of British Columbia REB. Approval from local institutional review boards (IRBs) was granted at each participating cohort site, including: Providence Health Care Research Institute Office of Research Services, University Health Network (UHN) REB, Ottawa Hospital REB, University of Toronto HIV REB, Women's College Hospital REB, Véritas IRB and Biomedical C REB of the McGill University Heath Centre (MUHC)

Written consent for study participation was obtained from all cohorts except: HAART Observational Medical Evaluation and Research Cohort (an information sheet is provided in lieu of a consent form; the IRB approves the retrospective use of anonymous administrative data without requiring consent); Ottawa Hospital Cohort, UHN and Maple Leaf Medical Clinic (IRB/ REBs approve the anonymous use of data retrospectively extracted from clinical databases without requiring consent); and MUHC and the Electronic Antiretroviral Therapy Cohort (patients sign a general waiver on starting a medical chart at the hospital, but no specific study-related consent is obtained; the IRB/REBs approve the anonymous use of data retrospectively extracted from clinical databases without requiring consent). 


\section{Publisher's Note}

Springer Nature remains neutral with regard to jurisdictional claims in published maps and institutional affiliations.

\section{Author details}

'Keenan Research Centre, Li Ka Shing Knowledge Institute, St. Michael's Hospital, Toronto, ON, Canada. 'Department of Family and Community Medicine, St. Michael's Hospital, Toronto, ON, Canada. ${ }^{3}$ University of Toronto, 410 Sherbourne Street, Toronto, ON ON M4X 1K2, Canada. ${ }^{4}$ Toronto General Research Institute, University Health Network, Toronto, ON, Canada. ${ }^{5}$ The Ottawa Hospital Research Institute, University of Ottawa, Ottawa, ON, Canada. ${ }^{6}$ British Columbia Centre for Excellence in HIV/AIDS, St. Paul's Hospital, Vancouver, BC, Canada. 'University of British Columbia, Vancouver, BC, Canada. ${ }^{8}$ Women's College Hospital Research Institute, Toronto, ON, Canada. ${ }^{9}$ Maple Leaf Medical Clinic, Toronto, ON, Canada. ${ }^{10}$ McGill University Health Centre, McGill University, Montreal, Quebec, Canada. ${ }^{11}$ Clinique Médicale I'Actuel, Montreal, Quebec, Canada. ${ }^{12}$ Regina Qu'Appelle Health Region, Regina, SK, Canada. ${ }^{13}$ Simon Fraser University, Burnaby, BC, Canada.

\section{Received: 1 November 2016 Accepted: 4 April 2017}

Published online: 11 April 2017

\section{References}

1. British HIV Association. British HIV Association guidelines for the treatment of HIV-1 positive adults with antiretroviral therapy 2015. Available at http:// www.bhiva.org/documents/Guidelines/Treatment/2015/2015-treatmentguidelines.pdf. Accessed 24 Jan 2016.

2. Panel on Antiretroviral Guidelines for Adults and Adolescents. Guidelines for the use of antiretroviral agents in HIV-1 infected adults and adolescents. Department of Health and Human Services. Available at http://www. aidsinfo.nih.gov/ContentFiles/AdultandAdolescentGL.pdf. Accessed 24 Jan 2016.

3. Shuter J. Forgiveness of non-adherence to HIV-1 antiretroviral therapy. J Antimicrob Chemother. 2008;61:769-73.

4. Zeldin RK, Petruschke RA. Pharmacological and therapeutic properties of ritonavir-boosted protease inhibitor therapy in HIV-infected patients. J Antimicrob Chemother. 2004:53:4-9.

5. Molina JM, Andrade-Villanueva J, Echevarria J, Chetchotisakd P, Corral J, David N, et al. Once-daily atazanavir/ritonavir compared with twice-daily lopinavir/ritonavir, each in combination with tenofovir and emtricitabine, for management of antiretroviral-naive HIV-1-infected patients: 96-week efficacy and safety results of the CASTLE study. J Acquir Immune Defic Syndr. 2010;53:323-32.

6. Orkin C, DeJesus E, Khanlou H, Stoehr A, Supparatpinyo K, Lathouwers E, et al. Final 192-week efficacy and safety of once-daily darunavir/ritonavir compared with lopinavir/ritonavir in HIV-1-infected treatment-naïve patients in the ARTEMIS trial. HIV Med. 2013;14:49-59.

7. Lennox JL, Landovitz RJ, Ribaudo HJ, Ofotokun I, Na LH, Godfrey C, et al. A phase III comparative study of the efficacy and tolerability of three nonnucleoside reverse transcriptase inhibitor-sparing antiretroviral regimens for treatment-naïve HIV-1-infected volunteers: a randomized controlled trial. Ann Intern Med. 2014;161:461-7.

8. Mugavero MJ, May M, Ribaudo HJ, Gulick RM, Riddler SA, Haubrich R, et al. Comparative effectiveness of initial antiretroviral therapy regimens: ACTG 5095 and 5142 clinical trials relative to ART-CC cohort study. J Acquir Immune Defic Syndr. 2011:58:253-60.

9. Cuzin L, Pugliese P, Allavena C, Katlama C, Cotte L, Cheret A, et al. Comparative effectiveness of first antiretroviral regimens in clinical practice using a causal approach. Medicine. 2015;95:e1668.

10. Johnston SS, Juday T, Esker S, Espindle D, Chu BC, Hebden T, et al. Comparative incidence and health care costs of medically attended adverse effects among U.S. Medicaid HIV patients on atazanavir- or darunavir-based antiretroviral therapy. Value Health. 2013;16:418-25.

11. Nishijima T, Hamada Y, Watanabe K, Komatsu H, Kinai E, Tsukada K, et al. Ritonavir-boosted darunavir is rarely associated with nephrolithiasis compared with ritonavir-boosted atazanavir in HIV-infected patients. PLoS One. 2013;8:e77268

12. Aberg JA, Tebas P, Overton ET, Gupta SK, Sax PE, Landay A, et al. Metabolic effects of darunavir/ritonavir versus atazanavir/ritonavir in treatment-naive, HIV type 1-infected subjects over 48 weeks. AIDS Res Hum Retrovir. 2012;28:1184-95.
13. Palmer A, Klein M, Raboud J, Cooper C, Hosein S, Loutfy M, et al. Cohort profile: the Canadian Observational Cohort Collaboration. Int J Epidemiol. 2011:40:25-32.

14. Gray RJ. A class of k-sample tests for comparing the cumulative incidence of a competing risk. Ann Stat. 1988;16:1141-54.

15. Fine JP, Gray RJ. A proportional hazards model for the subdistribution of a competing risk. J Am Stat Assoc. 1999;94:496-509.

16. Bartlett JW, Taylor JM. Missing covariates in competing risk analysis. Biostatistics. 2016;17:751-63.

17. Rubin DB. Inference and missing data. Biometrika. 1976;63:581-92.

18. Rubin DB. Multiple Imputation for Nonresponse in Surveys. New York: Wiley; 1987.

\section{Submit your next manuscript to BioMed Central and we will help you at every step:}

- We accept pre-submission inquiries

- Our selector tool helps you to find the most relevant journal

- We provide round the clock customer support

- Convenient online submission

- Thorough peer review

- Inclusion in PubMed and all major indexing services

- Maximum visibility for your research

Submit your manuscript at www.biomedcentral.com/submit 